\title{
Enfrentando resultados programa de ingeniería de sistemas de la Universidad Simón Bolívar con las pruebas saber pro
}

\section{Facing results systems engineering program at Universidad Simon Bolivar with Saber Pro test}

S. Jiménez, L Reyes, M Cañón

Keywords:

Test KNOW PRO, quality education, reading competition, competencies, average scores.
Palabras clave:

Prueba SABER PRO, calidad en la educación, competencia lectora, competencias, promedio de puntajes.

\begin{abstract}
It's main function of evaluation in education, guidance and support actions to improve quality through collection, analysis and interpretation of valid and reliable information. The application of systematic tests to quality allows for linking results, progress, evolution and maturity of the prospective student performance including its vision in the workplace. In Colombia a good benchmark for quality in education is to succeed in the knowledge test scores pro, made by the state and implemented by students of institutions of higher education.

therefore, the aim of this paper is to show how was the performance of students in the systems engineering program at the University Simon Bolivar in the tests with respect to the races there evaluated through statistical graphics, and that measures would be relevant in order to improve if they are failures compared to other institutions.
\end{abstract}

Es función principal de la evaluación en la educación, orientar y apoyar las acciones de mejoramiento de calidad mediante la obtención, análisis e interpretación de información valida y confiable. La aplicación de pruebas sistematizadas en busca de calidad permite establecer vínculos entre resultados, avances, evolución y madurez en el desempeño del futuro estudiante incluyendo su visión en el campo laboral. En Colombia un buen referente de calidad en la educación, es obtener resultados satisfactorios en los puntajes de las pruebas saber pro, realizada por el estado y desarrollada por los estudiantes de instituciones de educación superior.

Por esto, el objetivo del presente artículo, es mostrar cómo ha sido el desempeño de los estudiantes del programa de ingeniería de sistemas de la Universidad Simón Bolívar en dichas pruebas con respecto a las competencias allí evaluadas, por medio de gráficos estadísticos; y que medidas serian pertinentes para lograr mejorar si se encuentran fallas con respecto a otras instituciones del país. 


\section{INTRODUCCION}

A continuación se mostrara como han sido los resultados obtenidos de las pruebas saber pro del programa de ingeniería de sistemas del año 2009, 2010 y 2011 por medio de análisis de gráficos estadísticos, el cual se hará de forma sencilla y comprensible para el lector. En este caso se comienza citando las competencias lectoras, las cuales serán un punto de estudio para evaluar futuros profesionales de la facultad de ingeniería de sistemas, y un estado del arte de investigaciones previas relacionadas con la calidad en la educación, basándose en las pruebas realizadas por el estado (pruebas saber 11 y saber pro).

En Colombia, los Exámenes de Calidad de la Educación Superior (SABER PRO) para sistemas ya se ha unificado en el mismo grado de complejidad con otras carreras profesionales basados en una prueba genérica, son pruebas que sirven como herramienta de mejoramiento continuo, de acuerdo lo establecido a la ley 1324. [2]

Por esta razón se constituyen en uno de los mecanismos de evaluación de la calidad de los programas de las instituciones de educación superior.

\section{COMPETENCIAS}

La globalización y la modernización están creando un mundo cada vez más diverso e interconectado para comprender y desenvolverse bien en este pleno siglo, las personas necesitan por ejemplo, conocer $y$ dominar las tecnologías cambiantes y comprender enormes cantidades de información, por esta razón es de gran importancia hoy en día las competencias.

Tales tienen el objetivo de poner en marcha de forma integrada aquellos conocimientos, habilidades $y$ destrezas, tanto específicas como transversales, debe reunir un titulado para satisfacer plenamente las exigencias.

Las competencias son capacidades que la persona desarrolla en forma gradual y a lo largo de todo el proceso educativo son evaluadas en diferentes etapas. (ANUIES). [3]
El profesional en ingeniería sistemas se crea con una actitud capaz de identificar ordenar y explicar con argumentos en forma coherente, los principios, los conceptos y los problemas fundamentales del aspecto organizacional. También cumplir con un área de desempeño tal como Gestionar y administrar la información de una organización a través del análisis, modelamiento y procesamiento de la misma utilizando herramientas computacionales e informáticas, integrando todos los componentes en un sistema de información (hardware, software, redes, servicios, procesos y clientes).

\section{A. Competencias lectoras}

Al mencionar las competencias lectoras, nos referimos a la búsqueda de difundir correctamente la palabra escrita, es decir, producir oralmente la palabra escrita, con independencia de que se acceda o no a su significado.

Algunas asociaciones tales como la [IEA] Asociación Internacional para la Evaluación de Logros Educativos definen la competencia lectora como la capacidad de comprender y usar las formas del lenguaje escrito requeridas por la sociedad y/o valoradas por la persona. Posteriormente, con ocasión del estudio PIRLS en el año 2001, el equipo de investigación encargado del proyecto en Desarrollo de la Lectura hizo más compleja esta definición. [4]

Las competencias lectoras son un punto esencial para estudio de tipo descriptivo, de carácter longitudinal para que facultades tales como ingeniería de sistemas cuenten con las herramientas de conocimientos necesarias para poder afrontar problemas de ingeniería relacionados con la gestión, la organización y el manejo de la Información, basándose en fundamentos científicos, tecnológicos, técnicos y aplicados de las ciencias de ingeniería, de computación, administrativas y humanas, con el fin de que las organizaciones mejoren su productividad y competitividad, para optimizar los recursos físicos y financieros que está posea, con pensamiento crítico frente a cualquier situación y cabe mencionar optar por posiciones éticas. 


\section{ESTADO DEL ARTE}

La investigación que se está llevando a cabo, por parte de la universidad Simón Bolívar, tiene como finalidad realizar un análisis de las competencias que se ofrecen en el proceso de desarrollo del ingeniero de sistemas con lo que se evalúa en las pruebas del estado, esto en busca de una mejora en la calidad de la educación de los próximos profesionales.

Es de conocimiento de todos la necesidad que existe tanto nacional como internacionalmente, que los niveles educativos estén en una constante mejora, por lo tanto las instituciones que brindan formación a la población estudiantil, realizan investigaciones para indagar y descubrir cuáles son las debilidades o fallas que se presentan internamente, $y$, de esta manera tomar medidas pertinentes para poder alcanzar altos estándares de calidad.

A nivel local se ha tomado como referente el proyecto "FACTORES SOCIOECONÓMICOS DETERMINANTES DEL DESEMPEÑO ACADÉMICO UNIVERSITARIO: EL CASO DE LA PRUEBA ECAES -2009 EN LA REGIÓN CARIBE COLOMBIANA", desarrollado por Gustavo Rodríguez A. y Marco Ariza Dau en la Fundación Universidad del Norte, su propósito principal fue Analizar la influencia de los factores socioeconómicos en el rendimiento académico de los estudiantes Universitarios a los que se la aplicó la prueba ECAES en el año 2009 en la región Caribe colombiana.

La metodología que utilizaron los investigadores fue un enfoque Cuantitativo, teniendo en cuenta que se recurre a la medición estadística para hacer inferencia a partir de los resultados obtenidos. El tipo de estudio es Descriptivo - Explicativo, en la medida en que pretende describir los factores socioeconómicos de los estudiantes que fueron objeto de la prueba ECAES 2009, y establecer los factores socioeconómicos que inciden en los resultados obtenidos por los estudiantes que fueron participes de la prueba ECAES 2009.

El diseño es No Experimental, debido a que no se recurre a la manipulación de variables y los fenómenos se analizan en su estado natural. Además, es de Corte Transversal, dado que los datos utilizados corresponden a los resultados específicos de la prueba
ECAES - 2009. En este sentido, y dentro de este diseño se propone el Correlacional - Causal, en virtud de los análisis de relación de causa efecto entre dos o más variables que pueden hacerse en unos momentos determinados.

Las técnicas de análisis de datos utilizadas comprende dos niveles: El primero, es el análisis descriptivo de los datos, el cual se abordó con el uso de distribuciones de frecuencia y el empleo de análisis multivariado, concretamente el Análisis en Correspondencias Múltiples (ACM), donde intentaron establecer una tipología a partir de los factores socioeconómicos que caracterizan a los estudiantes universitarios de la región Caribe colombiana. El segundo nivel de análisis, comprendió la modelización econométrica del rendimiento académico como variable dependiente (puntaje del inscrito en el componente de la prueba) y los factores socioeconómicos como variables independientes o explicativas. En la exploración de las bases de datos del ICFES y concretamente los resultados ECAES 2009, y en el propósito de conseguir un modelo econométrico pertinente para el rendimiento académico explicado por los factores socioeconómicos de los estudiantes. [5]

Otro proyecto importante, tomado en cuenta para esta investigación es "ELEMENTOS DE CALIDAD DE LA EDUCACIÓN SUPERIOR EN COLOMBIA INGENIERÍA DE SISTEMAS CASO DE ESTUDIO: CON BASE EN RESULTADOS SABER PRO 2009", Desarrollado por Daniel Bogoya M. en la Universidad de Bogotá Jorge Tadeo Lozano.

Este proyecto se fundamento en analizar el estado de la calidad de nuestra educación superior y también explorar, si bien el aprendizaje alcanzado en una institución de educación superior podría estar signado por las condiciones socio-eco nómica y académica de los estudiantes. La metodología utilizada consiste en que "se partió de las bases de datos ftp://ftp.icfes.gov.co y se procedió a diseñar los indicadores, siguiendo dos caminos: Primero, se agruparon los estudiantes que presentaron la prueba SABER PRO, versión 2009, de acuerdo con el programa académico al que pertenecen y el estrato socioeconómico suministrado por ellos mismos en el 
formulario de inscripción, para luego establecer y comparar el promedio de puntaje alcanzado por cada grupo en cada institución, frente al universo total de población. Y segundo, considerando como unidad de análisis a cada estudiante para quien se disponen sus resultados en las pruebas SABER 11 y SABER PRO 2009". [6]

A nivel Nacional se ha tomado como referente el proyecto "COMPETENCIAS DEL LENGUAJE: RELACIONES ENTRE LA ESCUELA Y LAS PRUEBAS DE ESTADO" desarrollado por Ronald Andrés Rojas López en la Universidad Externado de Colombia, el cual tenía como propósito esencial Identificar las competencias del lenguaje evaluadas en cinco instituciones del D.C. y establecer su relación con los resultados de la Prueba ICFES- 2009.

La metodología que utilizó fue la Investigación Correlacional a partir de un estudio de caso, con un diseño No experimental, transaccional correlacional. Este tipo de diseño investigativo realiza una observación de fenómenos ya existentes sin llegar a intervenir de manera intencionada en las variables de estudio. Dicha observación se da en un contexto natural para luego pasar a un proceso de análisis. [7]

Otros proyectos importantes realizados en el país por prestigiosas universidades son: "DETERMINANTES INDIVIDUALES DE DESEMPEÑO EN LAS PRUEBAS DE ESTADO PARA EDUCACIÓN MEDIA Y SUPERIOR EN COLOMBIA" y "¿CUÁL ES LA BRECHA DE LA CALIDAD EDUCATIVA EN COLOMBIA EN LA EDUCACIÓN BÁSICA Y EN LA SUPERIOR?".

Este proyecto, fue realizado por Marly Tatiana Celis Gálvez, Juan Felipe Jaramillo Salazar y Oscar Andrés Jiménez Orozco en la Universidad de Manizales. Ellos identificaron la existencia y características de la brecha en la calidad de la educación (básica y superior), que se ofrece a individuos de distintos niveles socioeconómicos en Colombia. Para ello, utilizaron los resultados de las pruebas Saber 11 y Saber Pro como indicadores de la calidad de la educación básica y superior $y$, mediante la caracterización socioeconómica de los individuos que presentan las pruebas, establecieron cuáles segmentos de la población colombiana son discriminados en sus posibilidades vitales al recibir educación de menor calidad.

Estos investigadores plantearon un enfoque metodológico cuantitativo aplicado en tres etapas: la primera es la descripción de los datos, donde construyeron la tipología de los individuos que obtuvieron distintos niveles de rendimiento en las pruebas, según sus características socioeconómicas. En la segunda fase, los investigadores encontraron diferencias regionales en esas tipologías empleando regresiones multinivel sobre los puntajes de las pruebas. Finalmente, en la tercera etapa utilizaron la metodología de Propensity Score Matching para conocer si el desempeño en la educación básica y en la superior está asociado y el grado de correspondencia en las características socioeconómicas entre individuos de diferentes niveles de rendimiento de ambas pruebas, para saber el grado de discriminación en la calidad de la educación colombiana." [8]

EI proyecto "DETERMINANTES INDIVIDUALES DE DESEMPEÑO EN LAS PRUEBAS DE ESTADO PARA EDUCACIÓN MEDIA Y SUPERIOR EN COLOMBIA" fue realizado por Jaime Orjuela Viracacha en la Universidad Distrital Francisco José de Caldas, el objetivo de estudio de esta investigación fue analizar cuáles son los factores determinantes en el resultado de las pruebas de estado; para llevar a cabo esta investigación se partió como base de los datos obtenidos del Icfes y se utilizo una metodología donde se exploraba y comparaba tres enfoques de aprendizaje computacional para la selección de atributos y determinación de relevancia: El enfoque Bayesiano, El enfoque de árboles de clasificación y el de métodos kernel. Para compararlo con un método ampliamente aplicado al caso de interés que es el análisis de componentes principales, para aportar valor a los estudios anteriores sobre el tema. [9]

Ya que el ICFES promueve el uso, análisis y mejoramiento de la información que ese instituto genera, como producto de las evaluaciones adelantas a estudiantes de educación básica, media y superior en Colombia, muchas instituciones lo toman como referente para sus investigaciones, incluso 
instituciones de otros países como es el caso de la Universidad Complutense de Madrid que realizo una investigación basándose en estos datos, el proyecto lleva como nombre" ANÁLISIS DE LA EFICACIA DE APRENDIZAJE Y EFICACIA DE LAS INSTITUCIONES EDUCATIVAS MEDIANTE EL USO DE LOS DATOS DE LA PRUEBA CENSAL SABER 2009: APLICACIÓN DE LOS MODELOS DE ECUACIONES ESTRUCTURALES." Realizado por Maritza del Carmen Romero Duarte. Esta autora formuló, analizó y validó un modelo conceptual de relaciones causales de la eficacia educativa medidas a través del rendimiento de los estudiantes y de los factores asociados a éste, que fuera generalizable a las instituciones escolares del sector educativo colombiano. La metodología que se utilizo fue la modelización estructural aparece con el modelo LISREL (Jöreskog, 1970), del que surgen más adelante otros modelos derivados y similares.

Los Modelos de Ecuaciones Estructurales (MEE) contemplan dos modelos independientes pero relacionados. El primero de ellos, el modelo de medida que define la relación entre las variables observables y las variables latentes; consta de ecuaciones que especifican las 9 relaciones entre las variables latentes (factoriales), ya sean endógenas (dependientes) o exógenas (independientes) y variables manifiestas (u observables). $\mathrm{Y}$ el modelo estructural mide las relaciones entre las variables latentes y especifica cuáles de éstas influyen directa o indirectamente en los valores de las otras variables latentes, proporcionando los índices de ajuste de los datos a un modelo propuesto; modelo que se basa en la investigación teórica, donde el conocimiento que el investigador tenga de la teoría le permite dar una estructura a los datos, para luego contrastar la bondad de ajuste del modelo. A este análisis de relaciones estructurales lineales o no lineales también se le define como análisis causal.

En estos modelos pueden encajar multitud de variables, cuya combinación en bloques según un esquema teórico previo, contribuye a esclarecer las relaciones de dependencia causal que pueden existir, como plantea [10]. Cabe anotar que, el diseño y elaboración de modelos de análisis ha pasado de trabajar exclusivamente con variables observables y estructuras evidentes, al manejo de constructos no observables y variables latentes, correspondientes a estructuras subyacentes, que si bien menos aparentes, pueden ser detectadas a partir del análisis de una matriz de datos, de covarianzas o de correlaciones. [10]

Se han tomado estos proyectos mencionados anteriormente, por el significativo aporte a la investigación que se viene adelantando en la Institución, y por la importancia de tener en cuenta las pruebas realizadas por el estado como herramientas para medir, competencias y calidad. Pero adicionalmente, se puede percibir como las competencias adquiridas en los colegios influyen en la universidad y en los resultados de las pruebas saber pro.

\section{PRUEBAS SABER PRO}

A continuación se presenta el análisis de los resultados arrojados por las pruebas SABER PRO desde el año 2009 hasta el año 2011 realizadas por los estudiantes del programa de Ingeniería de sistemas de la Universidad Simón Bolívar. Estas estadísticas se obtuvieron de las base de datos proporcionadas por el icfes.

Primeramente, se mostrará el rendimiento interno de los estudiantes, por ejemplo: al clasificar los puntajes por genero se observa que el rendimiento de los hombres ha sido mayor en comparación con las mujeres, en el año 2009 la diferencia es de solo 2 puntos, los hombres con un promedio de puntaje de 98,82 y las mujeres con un 96,80 ; pero el rendimiento del género femenino desmejoro en gran manera en el año 2010 en comparación con el género masculino teniendo una diferencia de 8 puntos, obteniendo estos últimos un puntaje de 101,22 y las mujeres de 93,20(ver Fig. 1). 


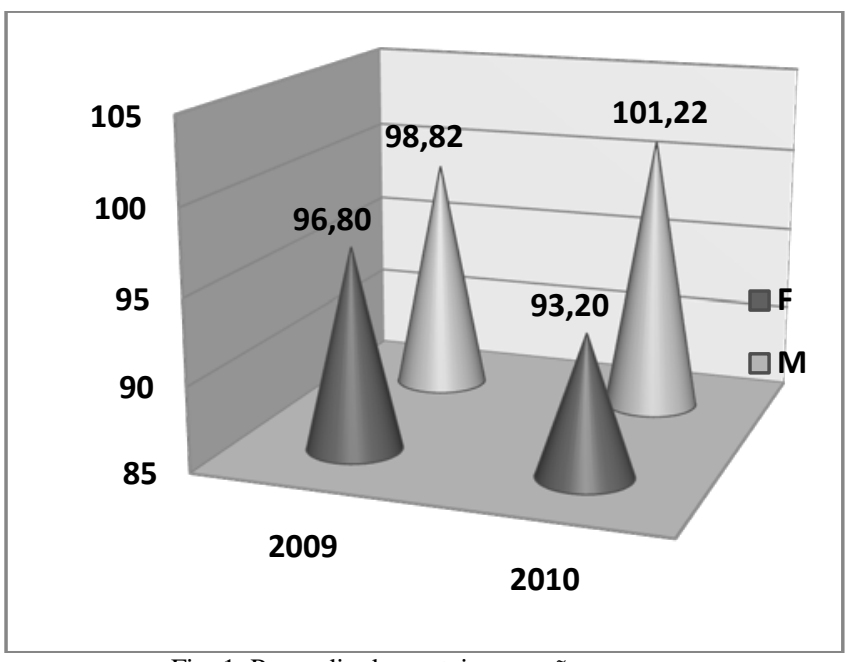

Fig. 1. Promedio de puntajes por año y genero

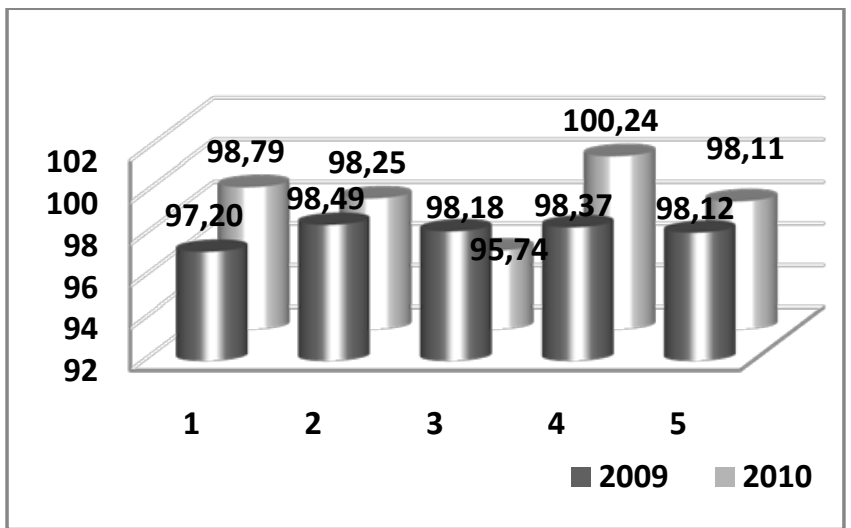

Fig. 2. Puntajes por año y estrato

En el año 2010 con respecto al resultado obtenido en el año 2009; por el contario los estudiantes de estrato 2,3 y 5 desmejoraron sus resultados del año 2010, teniendo en cuenta los puntajes que sacaron los estudiantes de estos mismos estratos en el año 2009.

Otro análisis que podemos sacar del grafico 3 anterior es que, los estudiantes de estrato 4 arrojaron los mejores resultados tanto en el año 2009 como en el año 2010.

Cuando se realiza la comparación entre género y estrato social, observamos que en todos los estratos de igual manera los hombres superan en el resultado del promedio a las mujeres como se muestra en la siguiente grafica.

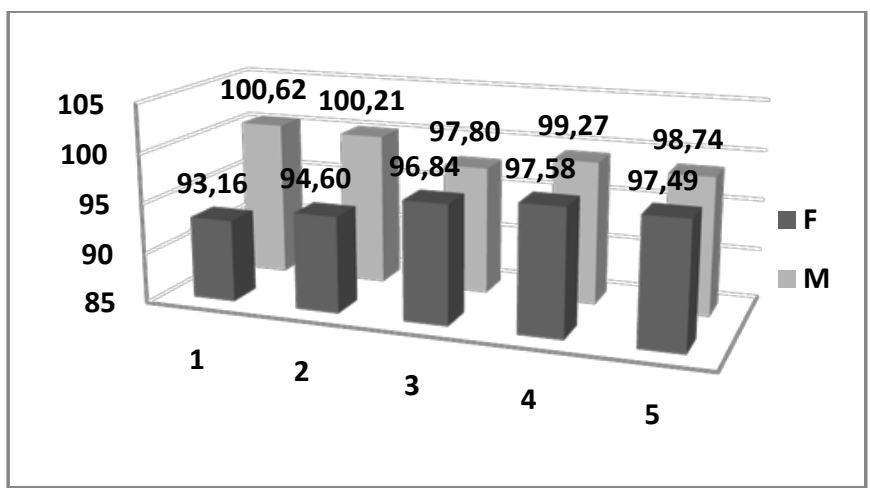

Fig. 3. Puntajes por género y estrato año 2009 y 2010

Este mismo análisis de estrato social es comparado por año realizado y observamos que, los estudiantes que pertenecen al estrato $1 \mathrm{y}$ al estrato 4 tuvieron una notable mejoría en el año Al momento de sacar estas estadísticas de las bases de datos, solo nos arrojo el promedio del programa de ingeniería de sistemas de la universidad Simón Bolívar de los años 2009 y 2010, y se comparo con el promedio obtenido a nivel nacional, como muestran las Fig. 4 y Fig. 5 . Y se puede observar como en estos años no se ha podido superar la media nacional, y aunque no estamos muy lejos de conseguirlo, todavía falta compromiso por parte de los estudiantes como de la institución, por no tomar las medidas pertinentes en el caso, porque no se trata de estar cerca de la media nacional, si no llegar a esta y sobrepasarla, ya que esto colocaría la universidad como una institución de alta calidad en la educación.

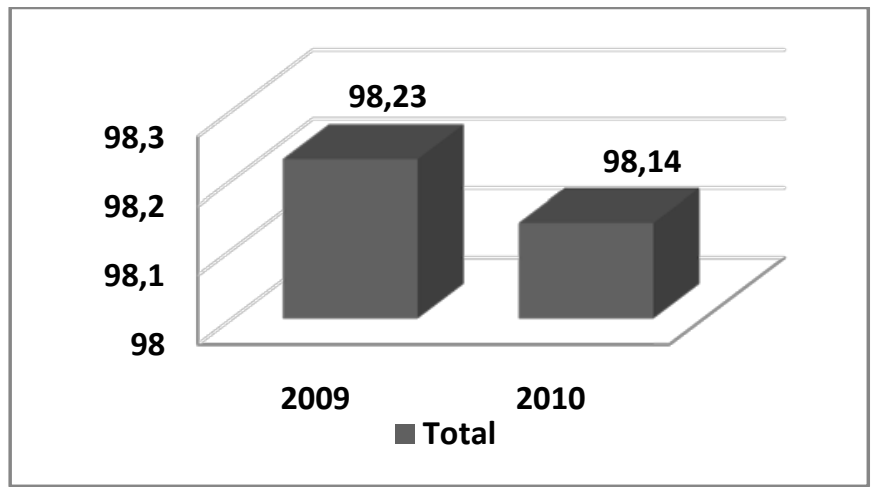

Fig. 4. Promedio Ing. de Sistemas de USB año 2009 y 2010 
PUNTAJE ECAES INGENIERÍA DE SISTEMAS 2006-2010

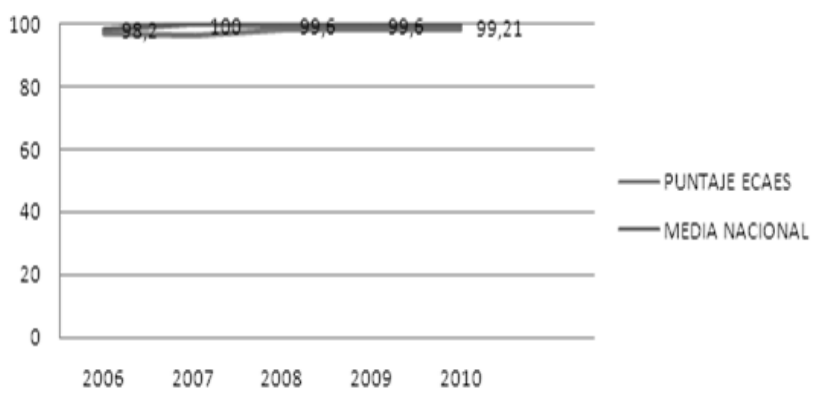

Fig. 5. Promedio Ing. de Sistemas nacional por año 206-2010

Luego de haber analizado el promedio de puntajes por distintas clasificaciones, nos centraremos en analizar cuál fue el desempeño que obtuvieron los estudiantes en las competencias evaluadas en las pruebas saber pro en el año 2011.

La fig. 6 nos muestra, los resultados de las pruebas saber pro de la competencia en comprensión lectora de 5 programas de la USB, y notamos que el programa de Ingeniería de Sistemas arrojo mejores resultados que los programas de Ing. Industrial y Admón. de Empresas, con un puntaje de 9,68; pero es superado por los programas de Contaduría Pública y Psicología, promedio que esperamos sea superado en el presente año, ya que la USB está fomentando en sus estudiantes utilizar los servicios que esta presta que ayuden a mejorar la comprensión de lectura como son APT, de igual manera incluyéndolos en programas de investigación para un mejor desarrollo en su formación.

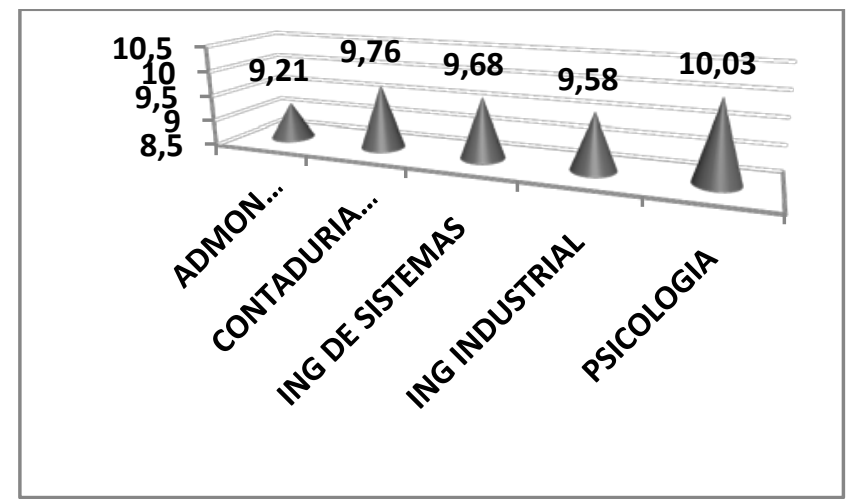

Fig. 6. Promedio de Competencia Lectora de 5 programas de USB año 2011
En el año 2011 las pruebas saber pro tomo 7 competencias a evaluar, que fueron comprensión lectora, comunicación escrita, solución de problemas, entender interpretar, inglés y pensamiento crítico.

Teniendo en cuenta esto, analizamos estos resultados de las competencias evaluadas de la USB (Fig. 7) con respecto a la media nacional (Fig. 8) de estas, y en ninguna de las competencias sobrepasamos la media nacional, pero en la competencia solución de problemas estamos bastante cerca con un promedio de 10,49 en comparación con la media que fue de 10,77 .

Por otra parte en ingles también estamos bastante cerca de la media, esto es muy importante, ya que en la formación como ingenieros el inglés es fundamental, porque es el lenguaje universal de los programas que se utilizan como herramienta de estudio y de trabajo.

Aunque hasta el momento, no han habido procesos de retroalimentación con los resultados de las Pruebas Saber Pro, para una mejoría notoria, esperamos que se tome conciencia tanto en los estudiantes, como profesores y directivas de la institución de la importancia de sobresalir y obtener excelentes resultados en estas pruebas para lograr posicionarnos como una gran institución de calidad.

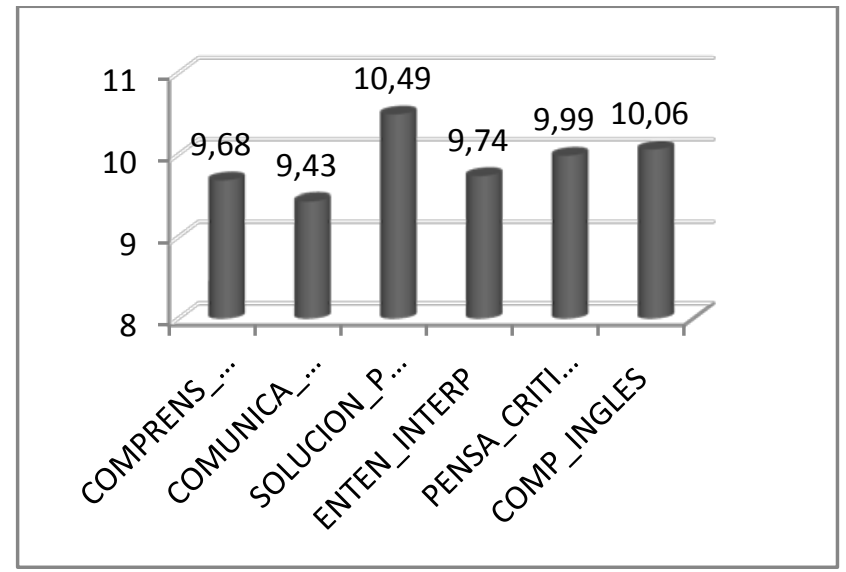

Fig. 7. Promedio por Competencias Ing. de Sistemas - USB año 2011 


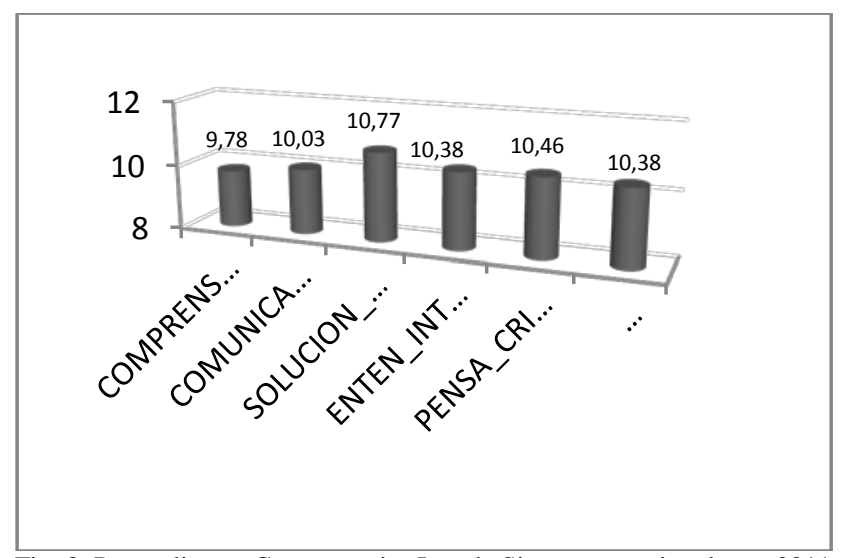

Fig. 8. Promedio por Competencias Ing. de Sistemas -nacional año 2011

\section{CONCLUSIONES}

En términos generales, el puntaje de las pruebas Ecaes- Saber Pro, obtenido por los estudiantes de ingeniería de sistemas se ha mantenido por encima del 95, notándose una mejoría al comparar los dos últimos años.

Los resultados de la pruebas del 2011 en adelante no son comparables con los años anteriores esto debido a que el icfes cambio los componentes evaluados, pero en esta última prueba el componente solución de problemas es el mejor evaluado, esto debido a que hace parte del núcleo común de ingeniería y a los programas institucionales de apoyo a esta área por parte de la universidad y del programa.

Sin embargo se debe seguir buscando nuevas estrategias para mejorar los resultados que en comparación con otros programas de la misma universidad no estamos rezagados.

\section{REFERENCIAS}

[1] Instituto de investigación y debate sobre la gobernanza (2007) http://www.institut-ouvernance.org/es/analyse/fiche-analyse-291.html

[2] Congreso República (2009) Ley 1324 del 13 de julio

[3] Icfes (2011) Estudio internacional del proceso en competencias lectoras http://www.dgb.sep.gob.mx/informacion_academica/curso_taller/materiales instructor/definicion_competencias

[4]Icfes

(2011)http://www.icfes.gov.co/pirls/index.php?option=com_content\&view= article\&id=4\&Itemid $=13$

[5] ICFES, 10 de Octubre de 2010. Convocatoria ICFES para Estudiantes de Maestría y Doctorado Extraído en Abril 04 de 2012 en

http://www.icfes.gov.co/investigacion/phocadownload/convocatorias/rodrig uez\%20gj.pdf
[6] Daniel Bogoya M., Decano Facultad de Ciencias Naturales e Ingeniería en la Universidad Jorge Tadeo Lozano, 28 de Marzo de 2011. ELEMENTOS DE CALIDAD DE LA EDUCACIÓN SUPERIOR EN COLOMBIA Extraído en Abril 4 de 2012 en http://utadeo.edu.co/facultades/naturales/ecaes/ingenieria_de_sistemas_2009 .pdf

[7] ICFES, 10 de Octubre de 2010. Convocatoria ICFES para Estudiantes de Maestría y Doctorado Extraído en Abril 4 de 2012 en

http://www.icfes.gov.co/investigacion/phocadownload/convocatorias/rojas ra.pdf

[8] ICFES, 10 de Octubre de 2010. Convocatoria ICFES para Estudiantes de Maestría y Doctorado Extraído en Abril 4 de 2012 en http://www.icfes.gov.co/investigacion/phocadownload/convocatorias/celis\% 20mt.pdf

[9] ICFES, 10 de Octubre de 2010. Convocatoria ICFES para Estudiantes de Maestría y Doctorado Extraído en Abril 4 de 2012 en http://www.icfes.gov.co/investigacion/phocadownload/convocatorias/orjuela \%20j.pdf

[10] Romero, M. del C. (2010). Análisis de la eficacia de aprendizaje y eficacia de las instituciones educativas mediante el uso de los datos de la prueba censal saber 2009: Aplicación de los modelos de ecuaciones estructurales. Extraído en abril de 2012 en http://www.icfes.gov.co/investigacion/phocadownload/convocatorias/romer o\%20mc.pdf 